Trauma Berufskrankh 2015 • [Suppl 2]:

17:307-314

DOI 10.1007/s10039-015-0020-x

Online publiziert: 31. März 2015

๑) Springer-Verlag Berlin Heidelberg 2015
S. Matschke · P. Krämer · K. Wendl · P.A. Grützner

Klinik für Unfallchirurgie und Orthopädie, BG Unfallklinik Ludwigshafen, Ludwigshafen, Deutschland

\section{Verletzungen der subaxialen Halswirbelsäule}

\section{Aktuelle Therapiestandards}

\section{Epidemiologie}

Wirbelsäulenverletzungen betreffen ca. $6 \%$ aller Frakturen und ereignen sich in einer Häufigkeit von 64/100.000 Einwohner. Mit zunehmender Verletzungsschwere steigt die Wahrscheinlichkeit von Wirbelsäulenverletzungen. Im Falle eines Polytraumas liegt bei jedem dritten Verletzten mindestens eine Wirbelsäulenverletzung vor. Die häufigste Ursache stellen hierbei Verkehrsunfälle dar [9]. Die Häufigkeit für eine Verletzung der Halswirbelsäule (HWS) ist signifikant erhöht bei Patienten mit zusätzlichem Schädel-HirnTrauma sowie bei fehlenden Abwehrreflexen des Patienten zum Zeitpunkt des Unfallereignisses [4, 6]. Die subaxiale HWS (Segmente C3-C7) ist zum Zeitpunkt eines Unfallgeschehens aufgrund ihrer hohen Gesamtbeweglichkeit und der Hebelwirkung des Kopfes im Verletzungsfall deutlich anfälliger für Frakturen, Subluxationen und Luxationen als der angrenzende starre Abschnitt der thorakalen Wirbelsäule. Aus diesem Grund ereignen sich im Abschnitt der HWS mehr als $75 \%$ aller Dislokationen und Luxationsverletzungen der gesamten Wirbelsäule. Frakturen sind im Bereich der subaxialen HWS eher bei C6 und C7 lokalisiert, während Luxationen und Subluxationen sich am häufigsten zwischen C5/C6 und C6/C7 ereignen $[4,6]$. Entsprechend einer Veröffentlichung von Morishita et al. [12] finden sich in diesen Segmenten auch die häufigsten degenerativen Veränderungen. Das Ausmaß der Verletzungsschwere im Bereich der subaxialen HWS variiert sehr und reicht von der häufigen einfachen Weichteilverletzung im Sinne einer Muskelzerrung oder Distorsion bis zur er- heblichen Frakturdislokation mit Spinalkanalverlegung. Ein Drittel der subaxialen HWS-Verletzungen, die im Rahmen einer großen Multicenterstudie [4] detektiert wurden, zeigten keine klinische Signifikanz. Trotz der Häufigkeit leichter Verletzungen im Bereich der subaxialen HWS sind gleichzeitig $55 \%$ aller traumatischen Querschnittslähmungen im zervikalen Wirbelsäulenabschnitt lokalisiert [18].

\section{Diagnostik}

Das primäre Ziel der diagnostischen Abklärung von HWS-Verletzungen ist das Erkennen oder der Ausschluss einer traumatischen substanziellen Schädigung. Diese kann rein ossär vorliegen und auch in Kombination mit einer ligamentären oder diskoligamentären Begleitverletzung. Ebenso kann eine isolierte diskoligamentäre Instabilität ohne begleitende Wirbelbruchverletzung bestehen.

\section{Konventionelle Röntgendiagnostik}

Bei entsprechendem Unfallmechanismus und Verdacht auf eine HWS-Verletzung stellt nach klinischer Untersuchung die konventionelle Röntgendiagnostik auch heute noch die Diagnostik der ersten Wahl dar.

Ist im sagittalen Strahlengang die anteriore bzw. posteriore vertebrale Linie, die spinolaminäre Linie oder die interspinöse Linie unterbrochen, ist dies immer Hinweis für eine Verletzung. Im Normalfall stellen sich diese Linien fortlaufend, leicht gebogen und ohne Unterbrechung dar (• Abb. 1). Gleichzeitig erfolgt die Beurteilung der Wirbelkörper selbst. Eine Verletzung der Hinterkante ist nur bei starker Dislokation zu erkennen. Die Beurteilung des tatsächlichen Ausmaßes und einer evtl. vorliegenden Spinalkanaleinengung ist erst durch ein Computertomogramm möglich [14].

Im frontalen Strahlengang geben ein vergrößerter interpedikulärer und interspinöser Abstand den entscheidenden Hinweis auf eine dorsale Zerreißung der ligamentären Strukturen im Falle einer Distraktionsverletzung. Bogenfrakturen sind ebenso häufig im frontalen Strahlengang zu erkennen.

Es ist unbedingt darauf $\mathrm{zu}$ achten, dass die gesamte HWS einschließlich des zervikothorakalen Übergangs dargestellt wird, d. h. bis Deckplatte Thl, da dieser Bereich eine hohe Verletzungsinzidenz aufweist [19].

Ist aufgrund anatomischer Bedingungen (z. B. adipöser Patient mit kurzem Hals) auch durch eine sog. Schwimmeraufnahme keine nativradiologische Darstellung des zervikothorakalen Übergangs möglich, muss dieser Abschnitt durch eine Computertomographie (CT) abgeklärt werden.

\section{Computertomographie}

Die CT erlaubt eine hochsensitive überlagerungsfreie Darstellung des Achsenskeletts. Die Spezifizität der Spiral-CT liegt bei $100 \%$, die Sensitivität bei $99 \%$ und die Wahrscheinlichkeit einer „missed spine injury“ bei 0,04\% [15].

Aufgrund der schnellen Verfügbarkeit der Mehrzeilen-Spiral-CT mit ihrem hohen Aussagewert kommt ihr heute die entscheidende Bedeutung in der Akutdiagnostik beim polytraumatisierten $\mathrm{Pa}$ tienten zu. Im Ergebnis ermöglicht die CT 


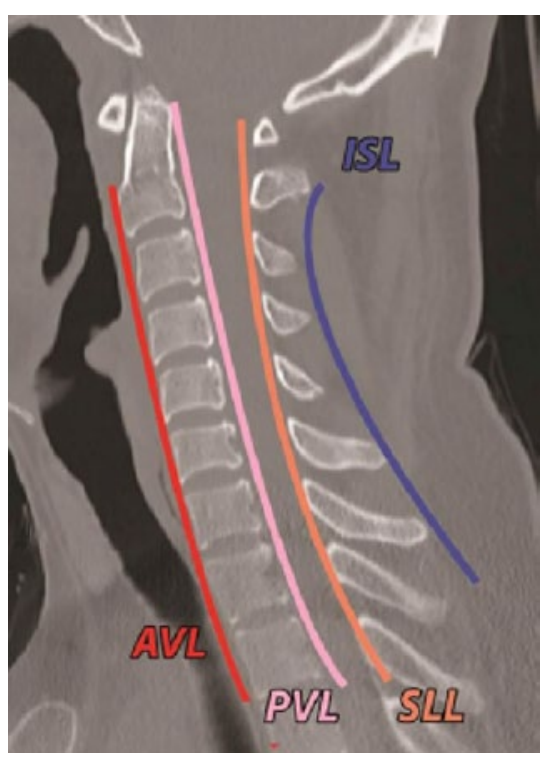

Abb. $1 \Delta$ Sagittales CT-Rekonstruktionsbild einer Halswirbelsäule. Rote Linie: anteriore vertebrale Linie (AVL), pinkfarbene Linie: posteriore vertebrale Linie $(P V L)$, orangene Linie: spinolaminäre Linie (SLL), blaue Linie: interspinöse Linie (ISL)

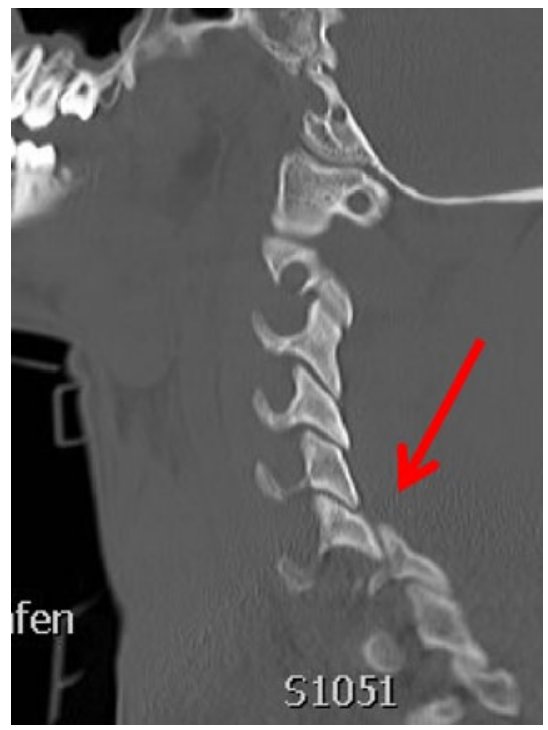

Abb. $2 \Delta$ Luxation der kleinen Wirbelgelenke C6/C7 (roter Pfeil)

eine unmittelbare exakte Beurteilung der Stellung der Wirbelsäule und des Frakturverlaufs. Mit diesem Verfahren wird das Ausmaß der Dislokationen von Hinterkantenfragmenten und einer resultierenden Spinalkanaleinengung genau ersichtlich. Eine Spinalkanalweite wird in den axialen Schnitten der CT gemessen. Die CT-Untersuchung erlaubt eine exakte Beurteilung der Stellung der kleinen Wirbelgelenke in Hinblick auf Lu-

\begin{tabular}{|c|c|c|c|c|}
\hline \multirow[t]{4}{*}{ A } & \multirow[t]{4}{*}{$\begin{array}{l}\text { Kompressions- } \\
\text { verletzungen }\end{array}$} & A1 Impaktionsbrüche & $\begin{array}{l}\mathrm{A} 1.1 \\
\mathrm{~A} 1.2 \\
\mathrm{~A} 1.3\end{array}$ & $\begin{array}{l}\text { Deckplattenimpression } \\
\text { Keilbruch } \\
\text { Wirbelkörperimpaktion }\end{array}$ \\
\hline & & A2 Spaltbrüche & $\begin{array}{l}\text { A2.1 } \\
\text { A2.2 }\end{array}$ & $\begin{array}{l}\text { sagittaler Spaltbruch } \\
\text { frontaler Spaltbruch }\end{array}$ \\
\hline & & & A2.3 & Kneifzangenfraktur \\
\hline & & $\begin{array}{l}\text { A3 Berstungsbrüche } \\
-\cdot-\cdot-\cdot-\cdot-\cdot-\cdot-\cdot--\end{array}$ & $\begin{array}{l}\mathrm{A} 3.1 \\
\mathrm{~A} 3.2 \\
\overline{\mathrm{A}} 3 \overline{3} . \overline{3}\end{array}$ & 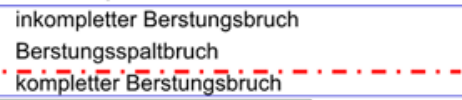 \\
\hline \multirow[t]{3}{*}{ B } & \multirow[t]{3}{*}{$\begin{array}{l}\text { Distraktions- } \\
\text { verletzungen }\end{array}$} & \begin{tabular}{|ll} 
B1 Flexiondistraktion \\
ligamentär
\end{tabular} & \begin{tabular}{|l|} 
B1.1 \\
B1.2
\end{tabular} & $\begin{array}{l}\text { mit Zerreissung des Diskus } \\
\text { mit Typ A-Fraktur }\end{array}$ \\
\hline & & $\begin{array}{l}\text { B2 Flexiondistraktion } \\
\text { ossär }\end{array}$ & $\begin{array}{l}\mathrm{B} 2.1 \\
\mathrm{~B} 2.2 \\
\mathrm{~B} 2.3\end{array}$ & $\begin{array}{l}\text { horizontale Zerreissung des Wirbels } \\
\text { Spondylolyse mit Bandscheibenzerreissung } \\
\text { Spondylolyse mit Typ A-Fraktur }\end{array}$ \\
\hline & & B3 Extensiondistraktion & $\begin{array}{l}\text { B3.1 } \\
\text { B3.2 } \\
\text { B3.3 }\end{array}$ & $\begin{array}{l}\text { Hyperextensionssubluxation } \\
\text { Hyperextensionsspondylolyse } \\
\text { hintere Luxation }\end{array}$ \\
\hline \multirow[t]{4}{*}{ C } & \multirow[t]{4}{*}{$\begin{array}{l}\text { Torsions- } \\
\text { verletzungen }\end{array}$} & $\begin{array}{ll}\text { C1 } & \text { Typ A-Fraktur mit } \\
& \text { Rotation }\end{array}$ & $\begin{array}{l}\text { C1.1 } \\
\text { C1.2 } \\
\text { C1.3 }\end{array}$ & $\begin{array}{l}\text { Impaktionsbrüche } \\
\text { Spaltbrüche } \\
\text { Berstungsbrüche }\end{array}$ \\
\hline & & $\begin{array}{ll}\text { C2 } & \text { Typ B-Fraktur mit } \\
& \text { Rotation }\end{array}$ & $\begin{array}{l}\mathrm{C} 2.1 \\
\mathrm{C} 2.2\end{array}$ & $\begin{array}{l}\text { B1- Läsion mit Rotation } \\
\text { B2- Läsion mit Rotation }\end{array}$ \\
\hline & & & C2.3 & B3- Läsion mit Rotation \\
\hline & & C3 Rotationsscherbruch & $\begin{array}{l}\text { C3.1 } \\
\text { C3.2 }\end{array}$ & $\begin{array}{l}\text { „Slice“ - Fraktur } \\
\text { Schrägfrakturen }\end{array}$ \\
\hline
\end{tabular}

Abb. $3 \Delta$ AO-Klassifikation von subaxialen HWS-Verletzungen in Anlehnung an Magerl et al. [11]

xationen ( $\mathbf{\bullet}$ Abb. 2) oder Subluxationen als Zeichen einer Distraktions- oder Rotationsdistraktionsverletzung.

Begleitende Verletzungen der A. vertebralis werden in der Kontrastmittelphase detektiert. Ein dringender Verdacht auf eine Verletzung der A. vertebralis und die Indikation für eine notwendige Abklärung bestehen insbesondere bei

- Facettengelenkdislokationen,

- Wirbelkörpersubluxationen oder

Wirbelkörperluxationen,

- Querfrakturen des Foramens der

A. vertebralis und

- Verletzungen der oberen HWS.

\section{Magnetresonanztomographie}

Die Beurteilung einer diskoligamentären Verletzung mit Bandscheibenrupturen und traumatische Bandscheibenhernien, des Interspinalraums und des Myelons ist die Domäne der Magnetresonanztomographie (MRT). Die Sensitivität für die Darstellung einer traumatischen Bandscheibenruptur liegt zwischen 79 und $93 \%$ [5].

Eine Unterbrechung im Verlauf des vorderen oder hinteren Längsbandes in Kombination mit der Darstellung der Verletzung der interspinösen Bänder und dorsaler Einblutungen ergibt den drin- genden Verdacht auf eine diskale Verletzung auch bei fehlendem MRT-Nachweis eines entsprechenden Flüssigkeitssignals in der STIR-Sequenz und den T2gewichteten Aufnahmen.

Die MRT-Diagnostik intraspinaler Verletzungen, wie Myelonkontusionen, Myeloneinblutungen und epidurale Hämatome, ist von besonderer Bedeutung bei neurologischen Ausfällen im Verletzungsfall, insbesondere bei unauffälligem CT-Befund (SCIWORA-Verletzung).

Perivertebrale Weichteil- und intravertebrale Wirbelkörperödeme geben Hinweise auf ein stattgefundenes relevantes Wirbelsäulentrauma, auch wenn direkte Frakturzeichen fehlen.

Des Weiteren ermöglicht eine MRAngiographie den Ausschluss oder Nachweis von Gefäßdissektionen oder intramuralen Blutungen. Im Vergleich zur Kontrastmittel-CT ist jedoch eine Differenzierung vorbestehender Plaques der Gefäßwand erschwert.

\section{Diagnostisches Vorgehen bei radiologisch unauffälligem Befund}

Besteht nach einem Trauma eine klinische Beschwerdesymptomatik ohne Nachweis einer Verletzung im Röntgenbild, ist zum Ausschluss einer diskoliga- 
mentären Verletzung eine weitere Diagnostik notwendig.

Hierfür bietet sich neben der MRT, die nicht immer zeitgerecht verfügbar ist, die Möglichkeit von Funktionsaufnahmen an. $\mathrm{Zu}$ unterscheiden sind hierbei Funktionsaufnahmen in Reklinations- und Inklinationsstellung im Röntgen (aktive Untersuchung) von passiv geführten Bewegungen unter Bildwandlerkontrolle (passive Untersuchung; [16]). Allerdings kann mit beiden Untersuchungstechniken durch eine Muskelanspannung des Patienten eine diskoligamentäre Verletzung u. U. nicht zur Darstellung kommen.

Bei weiterhin bestehendem Verdacht auf eine diskoligamentäre Verletzung und fehlendem Nachweis einer traumatischen Bandscheibenruptur in der initialen MRT-Untersuchung (die Sensitivität liegt zwischen 79-93\%) führen wir im Verlauf eine CT-Verlaufskontrolle zum Ausschluss einer evtl. auftretenden Subluxationsstellung (Beurteilung in den sagittalen Rekonstruktionen) durch.

\section{Klassifikation}

Eine der ersten Klassifikationen, basierend auf einer Analyse von über $1000 \mathrm{~Pa}$ tienten mit einer traumatischen Querschnittslähmung, veröffentlichte Sir Frank Holdsworth [8] im Jahre 1970.

Zwölf Jahre später stellten Ben Allen et al. [1] eine Klassifikation ausschließlich für die subaxiale HWS vor. Diese rein mechanistische Klassifikation findet heute noch im angloamerikanischen Sprachbereich eine breite Anwendung. In der Verletzungsschwere zunehmend werden 5 Hauptgruppen mit jeweiligen Untergruppen unterschieden: Verletzungen durch Flexion/Kompression (5 Subgruppen), reine Kompression (3 Subgruppen), Extension/Distraktion (4 Subgruppen), Extension/Kompression (5 Subgruppen), Extension/Distraktion (2 Subgruppen) und Lateralflexion (2 Subgruppen).

Ebenso im angloamerikanischen Sprachbereich verbreitet ist die 1986 veröffentlichte Harris-Klassifikation [7], die eine Unterteilung in 7 Hauptgruppen (Flexion, Flexion/Rotation, Extension/ Rotation, vertikale Kompression, Hyperextension, laterale Kompression, diverse nicht näher bezeichnete Mechanismen)

Trauma Berufskrankh 2015 - [Suppl 2]: 17:307-314 DOI 10.1007/s10039-015-0020-x

(c) Springer-Verlag Berlin Heidelberg 2015

S. Matschke · P. Krämer · K. Wendl · P.A. Grützner

Verletzungen der subaxialen Halswirbelsäule. Aktuelle Therapiestandards

\section{Zusammenfassung}

Klassifikation. Zur subaxialen Halswirbelsäule wird der Abschnitt ab dem Bandscheibenraum $\mathrm{C} 2 / \mathrm{C} 3$ bis in den zervikothorakalen Übergang (C7/Th1) gerechnet. Grund für die Unterteilung in Verletzungen der subaxialen HWS und solche der oberen HWS ist der einheitliche anatomische Aufbau unterhalb von C2 mit hieraus gleich wirkenden biomechanischen Grundprinzipien für die einzelnen Bewegungssegmente. Entsprechend ergibt sich eine für alle Verletzungen unterhalb von $\mathrm{C} 2$ ähnlich anzuwendende Therapieoption.

Therapie. Im Gegensatz zu den Verletzungen der oberen HWS steht bei der Behand- lung von traumatischen subaxialen Instabilitäten ein primär ventrales Vorgehen im Vordergrund. Dies gilt auch für die Reposition von Subluxationen oder Luxationsverletzungen. Nach einem ventral fusionierenden Eingriff ist ein kombiniertes ventrodorsales Vorgehen erforderlich, wenn eine höhergradige posteriore Instabilität verbleibt.

Schlüsselwörter

Halswirbel · Gelenkinstablitität · Luxation . Wirbelbrüche · Chirurgisches Vorgehen

\section{Injuries of the subaxial cervical spine. Current standards in therapy}

\section{Abstract}

Classification. Injuries of the cervical spine can be categorized into subaxial injuries and injuries of the upper cervical spine. The subaxial section extends from segments $\mathrm{C} 2 / \mathrm{C} 3$ to segments C7/Th1 distally. The reason for this subclassification into upper cervical spine injuries and subaxial injuries is the uniform anatomical morphology from C2 down to Th1. The comparable anatomy of these segments is also reflected in the comparable biomechanics of these segments leading to similar therapeutic options.

Therapy. In contrast to the upper cervical spine, the therapeutic management of the subaxial spine is characterized primarily by ventral procedures. Traumatic instabilities and subluxations or luxations can usually be approached ventrally. In cases of persisting instabilities or relevant posterior instabilities a combination of ventral and dorsal approaches can be necessary.

\section{Keywords}

Cervical vertebrae - Joint instability .

Dislocation · Spinal fractures · Surgical procedures mit entsprechenden Untergruppen vornimmt.

Einen neuen Weg der Klassifikation von subaxialen HWS-Verletzungen beschritten Vaccaro et al. 2007 mit der Subaxial Cervical Spine Injury Classification (SLIC [20]). Ziel dieser Klassifikation ist die Ableitung der Therapie durch ein Punktesystem, das sich aus der Summe von 3 Hauptgruppen errechnet:

- der Frakturmorphologie,

- dem diskoligamentären Komplex und

- dem neurologischen Status.

Basierend auf der Veröffentlichung von Magerl et al. [11] wird heute im europäischen Sprachbereich die von der Arbeits- gemeinschaft für Osteosynthesefragen (AO) verbreitetete Klassifikation verwendet, die 3 Hauptgruppen unterscheidet:

- Typ A: Kompressionsverletzungen

- Typ B: Distraktionsverletzungen

- Typ C: Torsionsverletzungen

Die Hauptgruppen werden in jeweils 3 Untergruppen unterteilt, wobei jede Untergruppe nochmals in Subguppen differenziert werden kann (• Abb. 3).

Eine neue methologisch validierte Klassifikation von subaxialen HWS-Frakturen, angelehnt an die Magerl-Klassifikation mit Typ-A-, -B- und -C-Verletzung und 9 Untergruppen, wird gegenwärtig von einem internationalen Arbeitsteam überprüft und erstellt [17]. 

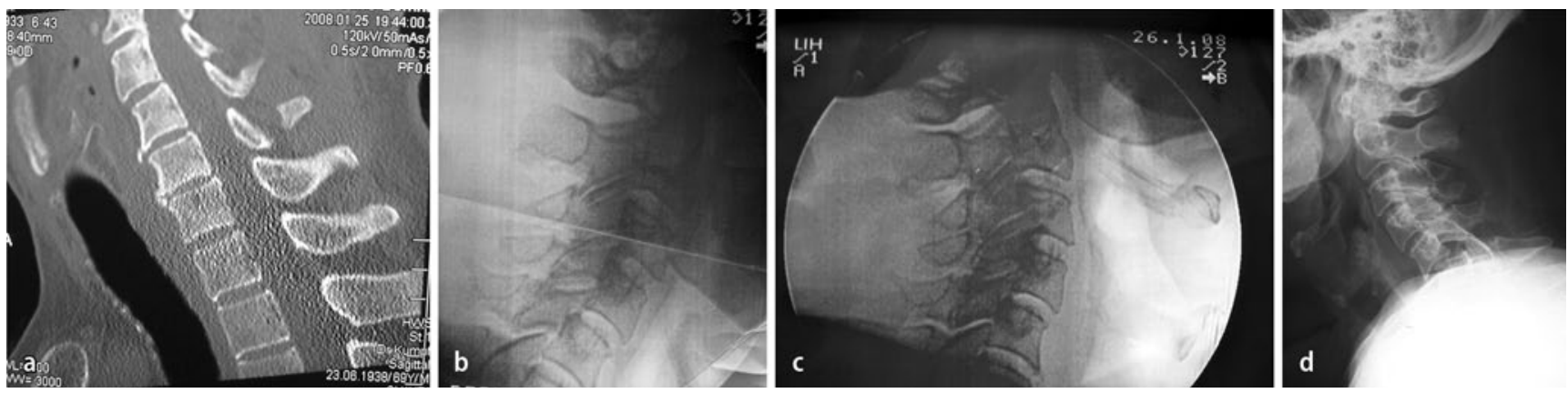

Abb. 4 a Sagittale CT-Rekonstruktion, regelrecht verlaufende AVL, VL, SSL, ISS bei einem 70-jährigen Patienten nach einem Verkehrsunfall. Im CT keine Verletzung darstellbar. AVL anteriore vertebrale Linie, PVL posteriore vertebrale Linie, SLL spinolaminäre Linie, ISL interspinöse Linie b,c Durchleuchtungsbilder passiv mit Reklination und Inklination zum Ausschluss einer diskoligamentären Instabilität ohne Verletzungshinweis, d Verlaufsbild nach 10 Tagen ohne neues Unfallereignis mit Darstellung einer ligamentären Instabilität mit Diskuszerreißung und Luxation C5/C6 Typ B1.1, keine motorischen Lähmungen

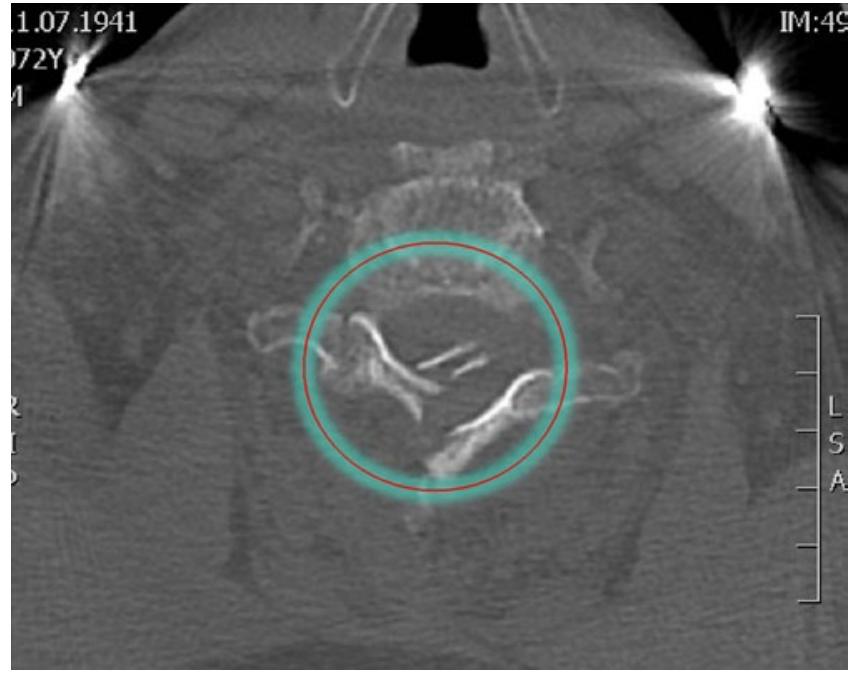

Abb. $5<$ Dislozierte Bogenfragmente mit Kompression des Myelons

\section{Therapieentscheidung}

Bei den subaxialen HWS-Verletzungen werden Impaktionsbrüche (Typ A1) und Spaltbrüche (Typ A2) der konservativen Behandlung zugeführt. Eine klare, evidenzbasierte Trennlinie zwischen konservativer und operativer Behandlung gibt es nicht. Da die Reststabilität gegen eine axiale Belastung im HWS-Abschnitt bei inkompletten Berstungsbrüchen (Typ A3.1) bzw. Berstungsspaltbrüchen (Typ A3.2) im Gegensatz zu Verletzungen der Brustund Lendenwirbelsäule deutlich höher ist, behandeln wir diese Verletzung primär konservativ. Ausnahmen stellen kyphotische segmentale Fehlstellungen von mehr als $15^{\circ}$ dar.

$\mathrm{Ab}$ den kompletten Berstungsbrüchen (Typ A3.3) überwiegt die Entscheidung zur Fusionsoperation aufgrund der mangelnden Reststabilität gegen eine axiale
Belastung ( $\bullet$ Abb. 3, rot gestrichelte Linie).

Bei allen Verletzungen des Typs B und $C$ besteht die Indikation zur operativen Therapie.

In unserem Patientengut war bei den Distraktionsverletzungen ein gehäuftes Vorkommen der Flexiondistraktion ligamentär mit Zerreißung des Diskus (Typ B1.1) sowie der Verletzungen des Typs B3 (• Abb. 3, grüne Umrandung) zu beobachten. Bei den Torsionsverletzungen fand sich in unserem Patientengut ein deutliches Überwiegen von TypC2-Verletzungen (Typ-B-Fraktur mit Rotation).

\section{Konservative Behandlung}

Alle Verletzungen der subaxialen HWS mit einer verbliebenen Reststabilität gegen Distraktions- und Rotationskräfte und ohne relevante sagittale Fehlstellung können einer konservativen Therapie zugeführt werden.

Entsprechend der Magerl-Klassifikation (-Abb. 3) handelt es sich hierbei um Verletzungen des Typs A1, A2 sowie des inkompletten Berstungstyps (A3.1). Maßgebend für die Entscheidung zu einer konservativen Therapie ist bei diesen Verletzungstypen, dass im sagittalen Profil keine kyphotische Fehlstellung größer als $15^{\circ}$ vorliegt, gemessen im bisegmentalen Winkel unter Einschluss der verletzten Bandscheibe.

Typisch für diese „stabilen“ Verletzungen ist die im kurzfristigen Verlauf mit einer entsprechenden Immobilisation der HWS abklingende Schmerzsymptomatik.

Die konservative Therapie wird in diesen Fällen durch eine äußere Ruhigstellung der HWS mittels Zervikalstütze (z. B. Philadelphia-Kragen, oder Vista collar ${ }^{\circledR}$, Aspen) durchgeführt. Eine Immobilisation erfolgt in diesen Fällen je nach Verletzungstyp über einen Zeitraum von 4 bis 6 Wochen.

Der Zervikalstütze kommt hierbei v. a. die Bedeutung einer „ermahnenden" Ruhigstellung vor Kopfbewegungen für einen begrenzten Zeitraum zu. Neben einer medikamentösen analgetischen Therapie dient sie auch der Vermeidung von Schmerzen durch muskuläre Verspannungen.

Keinesfalls kann eine höhergradige Instabilität der subaxialen Halswirbelsäule, auch bei regelrecht angepasster Zervikalstütze, ohne relevanten Korrekturverlust oder Gefahr einer translatorischen Dislokation zur Ausheilung gebracht werden. 

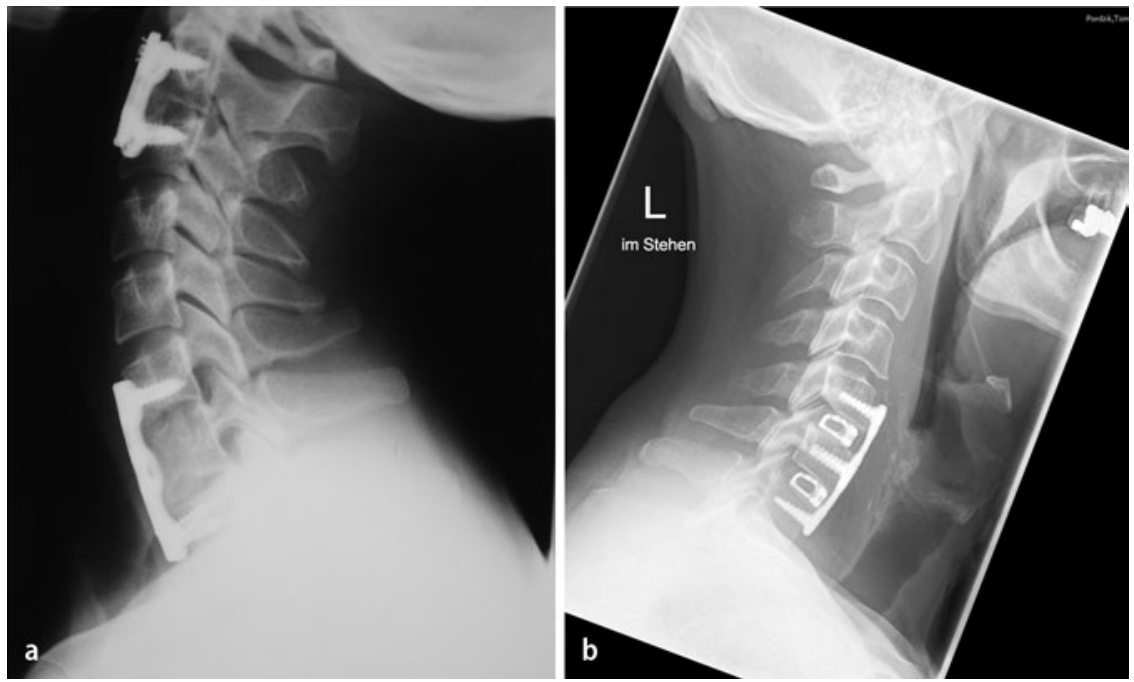

Abb. $6 \Delta$ a Monosegmentale Spondylodese C2/C3, bisegmentale Spondylodese C6/Th1 mit Beckenkammblock und winkelstabiler Plattenosteosynthese (CSLP ${ }^{\circledR}$, DePuy Synthes). b Ventral bisegmentale Spondylodese C5/C7 mit SynCage ${ }^{\circledR}$ (DePuy Synthes) und winkelstabiler Plattenosteosynthese (Skyline ${ }^{\circledR}$-Platte, DePuy Synthes)

Da diskoligamentäre Verletzungen und ihre Instabilitäten in der initialen Diagnostik unter bestimmten Umständen nicht sofort erkennbar sind, kann es in dieser Phase zu einer falschen Stabilitätsbeurteilung kommen. Deshalb ist eine Überprüfung der Stabilität nach Einleitung der konservativen Therapie durch kurzfristige radiologische Verlaufskontrollen erforderlich. Diese empfehlen wir nach 4, 7 und 11 Tagen sowie im weiteren Verlauf nach 3 Wochen (• Abb. 4).

Eine unerwartet neu aufgetretende Schmerzsymptomatik ist Anlass für eine aktuelle Bildgebung.

\section{Operative Behandlung}

\section{Zugangswege zur operativen Stabilisierung}

Zur operativen Versorgung von instabilen Halswirbelverletzungen ergibt sich die Option eines anterolateralen und eines dorsalen Vorgehens.

\section{Anterolateraler Zugang}

Der anterolaterale Zugang ist indiziert für alle Verletzungen, bei denen die Pathologie von ventral erreichbar ist, $d$. h. alle Instabilitäten durch eine verletzte/zerrissene Bandscheibe und/oder instabile Wirbelkörperfrakturen oder wenn eine Dekompression des Spinalkanals von ventral erforderlich ist.
Dieser Zugang verläuft entlang anatomisch definierter Strukturen und ist als atraumatisch zu bezeichnen, da keine Muskulatur abgelöst werden muss. Leitstruktur ist der Vorderrand des M. sternocleidomastoideus in entsprechender Verletzungshöhe. Ob der Zugang von der rechten oder linken Seite gewählt wird, sollte abhängig von der Händigkeit des Operateurs entschieden werden. So empfiehlt sich für einen rechtshändigen Operateur der Zugang von rechts. Ebenso ist bei monosegmentalen Versorgungen auch eine quere Hautinzision in Verletzungshöhe möglich.

Muss der Abschnitt C2/C3 von ventral erreicht werden, wird der Hautschnitt quer in einer Halsfalte unterhalb des Unterkiefers gelegt, als Leitstruktur dient lateral der Vorderrand des M. sternocleidomastoideus, kranial die Glandula submandibularis. Unterhalb von C3 bis Thl/ Th2 empfiehlt sich der Hautschnitt am Vorderrand des M. sternocleidomastoideus.

Auch für alle luxierten Verletzungen der subaxialen HWS ( $\bullet$ Abb. 4d) bietet sich das initiale operative Vorgehen von ventral an.

\section{Dorsaler Zugang}

Der dorsale Zugang ist immer verbunden mit der Durchtrennung und Ablösung der Muskulatur von den Processi spinosi und den Wirbelbögen zur Freilegung der
Facettengelenke. Im Vergleich zum anterolateralen Zugang ist keine Präparation in vorgegebenen anatomischen Spatien möglich. Indiziert ist das dorsale Vorgehen zweizeitig nach einer ventralen Spondylodese mit verbleibender dorsaler Instabilität. Da die heutigen winkelstabilen Schrauben-Stab-Systeme technisch relativ einfach unter Verwendung der Massa lateralis eingebracht werden können, um das biomechanisch erforderliche dorsale Zuggurtungsprinzip wiederherzustellen, verwenden wir das kombinierte ventrodorsale Vorgehen entsprechend der Indikation bei Distraktions- und Rotationsdistraktionsverletzungen. Der Vorteil ist die Möglichkeit einer funktionellen, sicheren Nachbehandlung ohne äußere Schienung durch einen Kragen.

Ein primär dorsales Vorgehen wird in den seltenen Fällen gewählt, bei denen eine Dekompression des Spinalkanals durch dislozierte Knochenfragmente von dorsal erforderlich ist, sowie bei langstreckigen, mehrsegmentalen hochinstabilen Verletzungen [3].

Verhakte Luxationsfrakturen stellen nur in Ausnahmefällen eine Indikation für ein primär dorsales Vorgehen dar. Eine suffiziente Reposition und Dekompression lassen sich in aller Regel von ventral erreichen.

Die Ziele der operativen Therapie bestehen in der

- Dekompression neurologischer

Strukturen

- Wiederherstellung der Stabilität

- Korrektur von Fehlstellungen

Bis auf Ausnahmefälle, bei denen eine Kompression neurogener Strukturen von dorsal vorliegt (•Abb. 5), erfolgt eine erforderliche Dekompression über ein ventrales Vorgehen. Die Wiederherstellung der Stabilität und die Korrektur von Fehlstellungen können sowohl über ein ventrales als auch dorsales Operationsverfahren durchgeführt werden.

\section{Ventrale Spondylodese}

Cloward [2] beschrieb die ersten ventralen Verfahren für eine Spondylodese der subaxialen HWS. Mit dem Aufkommen von winkelstabilen Implantaten und deren besserer Stabilität $[10,13]$ wurde die 

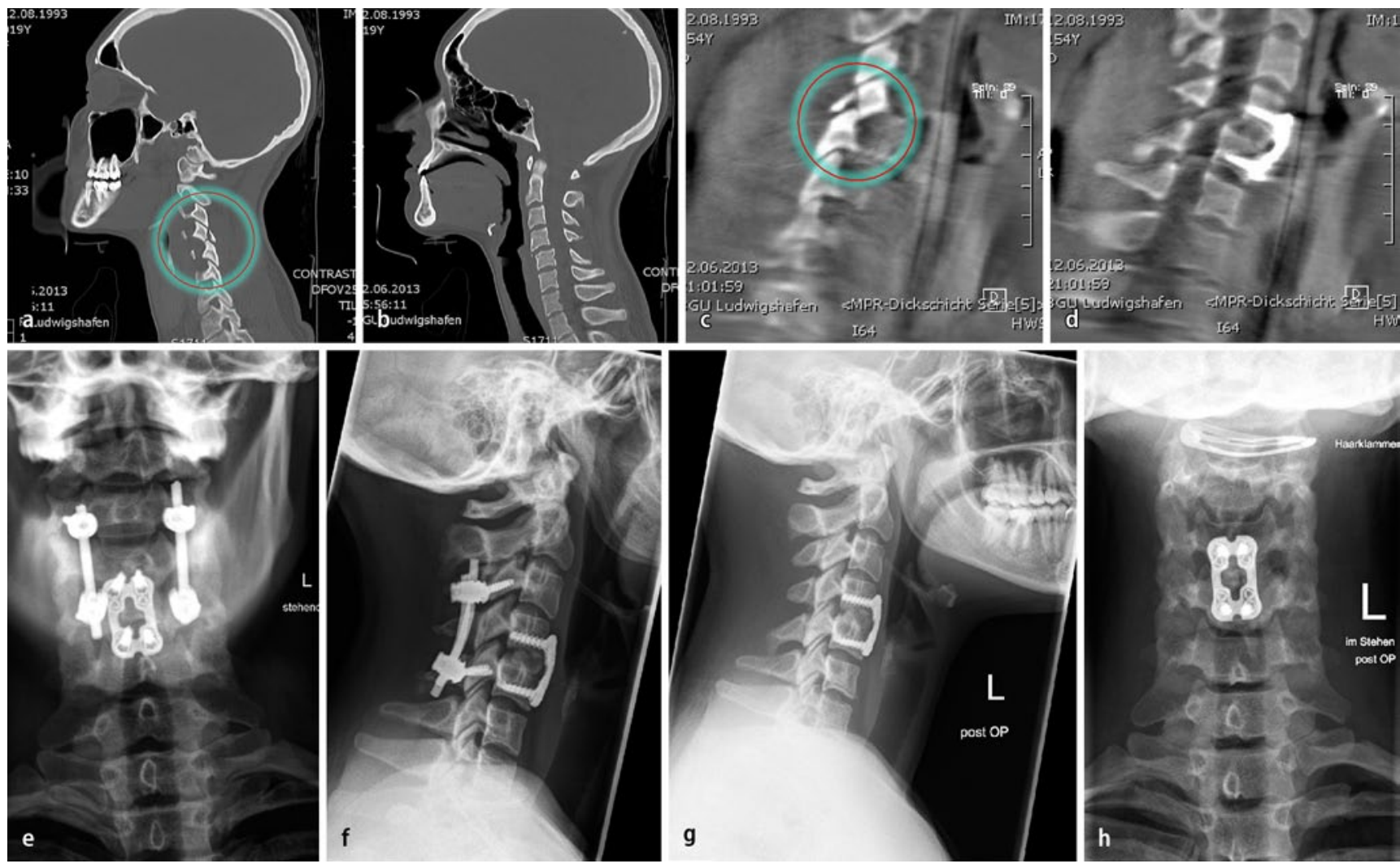

Abb. $7 \Delta$ a,b Distraktionsverletzung C5/C6 Typ B1.1 mit Subluxationsstellung der Facettengelenke. c,d Ventrale Spondylodese monosegmental, keine Reposition der Subluxationsstellung. e,f Zweizeitige dorsale Reposition und Instrumentierung über Massa-lateralis-Schrauben C3 nach C5. g,h Entfernung des dorsalen Fixateurs nach 6 Monaten

ventrale Spondylodese durch die Verwendung von polyaxialen Schrauben sicherer und einfacher mit weniger Schraubenlockerungen und Implantatversagen im Traumafall.

Der Zugang zur ventralen Spondylodese wird durch die Lagerung des Kopfes auf einer am Operationstisch befestigten, frei einstellbaren Kopflagerungsschale erleichtert. Präoperativ wird unter Durchleuchtung die Wirbelsäule entsprechend den physiologischen sagittalen Alignements gelagert. Besteht eine verhakte Luxation, wird unter Durchleuchtung beim relaxierten Patienten in Extension und leichter Flexion die Luxationsstellung der kleinen Wirbelgelenke reponiert. In der Mehrzahl der Fälle ist die dorsale ligamentäre Instabilität so groß, dass die Reposition ohne größere Manipulationsmaßnahmen erreicht wird. Präoperativ muss anhand der Schnittbilddiagnostik (CT/MRT) ausgeschlossen worden sein, dass durch das Repositionsmanöver rupturierte Bandscheibenanteile zusätzlich in Richtung des Spinalkanals verlagert wer- den können und zu einer zusätzlichen Myelonkompression führen.

Ob die ventrale Spondylodese mono-, bi- oder mehrsegmental erfolgt, hängt vom Verletzungstyp ab. Isolierte ventrale diskale Instabilitäten entsprechend den Typ-B2-Distaktionsverletzungen sowie instabile Verletzungen in Kombination mit einem inkompletten Berstungsbruch (Typ A3.1) bzw. einem Berstungsspaltbruch (Typ A3.2) können durch eine ventrale monosegmentale Fusion zur Ausheilung gebracht werden.

Nach Entfernung der rupturierten Bandscheibe und der instabilen Wirbelkörperanteile wird der Defekt entweder mit einem Beckenkammblock oder einem konfektionierten Cage aufgefüllt (• Abb. 6b). Eine Press-fit-Technik ist Voraussetzung für eine sichere Einheilung des Knochentransplantats.

Winkelstabile Plattensysteme dienen der Sicherung der Einheilung des Knochentransplantats sowie der Sicherung bis zum Eintreten der Fusion nach Einbringung eines Cages (• Abb. 6). Hierbei ist zum Schutz vor Anschlussdegenerationen auf eine exakte Lage der Platte zu achten; diese sollte das nächste gesunde Bandscheibenfach nicht tangieren.

\section{Kombiniertes ventrodorsales Vorgehen}

Verbleibt auch unter Verwendung winkelstabiler Implantate nach ventraler Spondylodese eine posteriore Instabilität, sehen wir die Indikation für eine zusätzliche dorsale Instrumentierung mit einem winkelstabilen Schrauben-Stab-System. Biomechanisch wird durch die dorsale Zuggurtung mit dem Fixateur das ,tension band principle" wiederhergestellt.

Als radiologische Zeichen nach ventraler Spondylodese gelten hierfür noch distrakt stehende Facettengelenke oder eine Subluxation der kleinen Wirbelgelenke (-Abb. 7a-d). Auch bei Distraktions- oder Rotationsverletzungen mit initialer Luxation der kleinen Wirbelgelenke sehen wir die Indikation für eine ventrodorsale Versorgung $(\bullet$ Abb. 7e,f). Der 

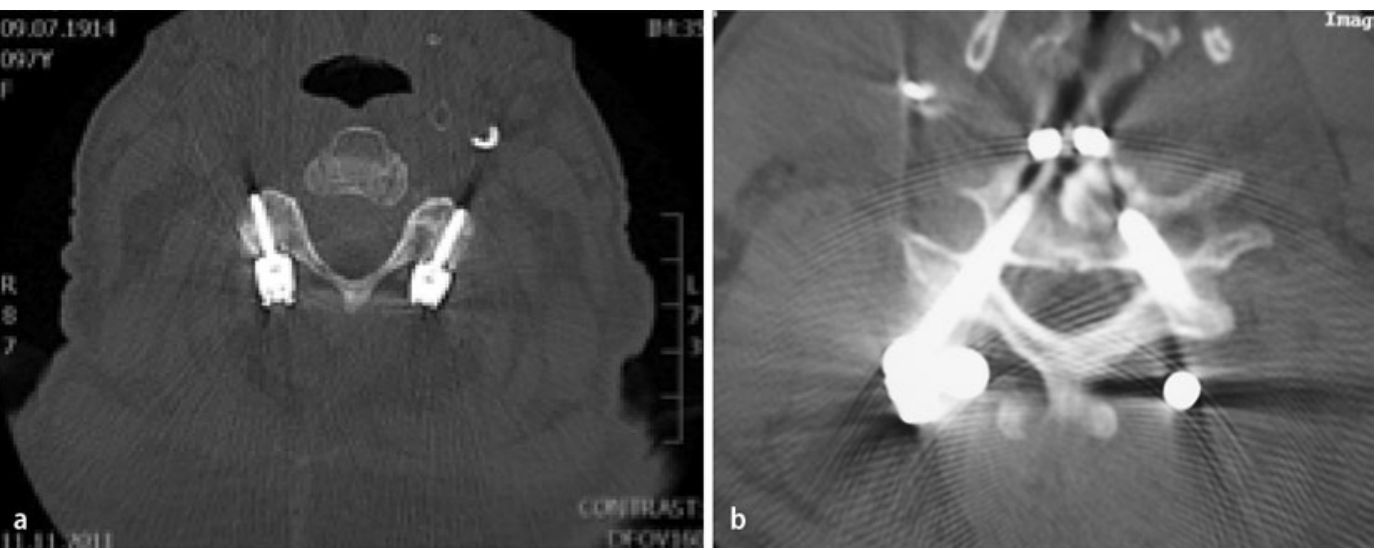

Abb. $8<$ a Massa-lateralisSchrauben, $\mathbf{b}$ transpedikuläre Schraubenlage C4

Vorteil ist eine primäre Belastungsstabilität der Wirbelsäule, die eine funktionelle Nachbehandlung ohne Zervikalstütze ermöglicht. Implantatlockerungen oder Korrekturverluste nach ventraler Spondylodese bei diesen hochinstabilen Verletzungen, die auch unter der Nachbehandlung mit einer Zervikalstütze auftreten können, lassen sich so sicher vermeiden.

Nach eingetretener Fusion empfehlen wir bei jungen Patienten eine frühzeitige Metallentfernung des Fixateurs nach 6 Monaten, um eine größtmögliche Funktion der Wirbelsäule zu erhalten

(- Abb. 7g,h).

Eine dorsale Schraubenplazierung erfolgt im Bereich der Massa lateralis bzw. bei C7 im Processus transversus (- Abb. 8a). Die transpedikuläre Schraubenlage ( $\bullet$ Abb. 8b) bietet den größtmöglichen Schraubenhalt für eine Instrumentierung. Im Vergleich zur Besetzung der Massa-lateralis-Schrauben ist dieses Verfahren jedoch technisch sehr anspruchsvoll und bleibt insofern Ausnahmeindikationen vorbehalten, wie z. B. instabile Distraktionsverletzungen bei Patienten mit Morbus Bechterew, bei denen eine ventrale additive Abstützung nicht möglich ist.

\section{Fazit}

- Anhand einer einheitlichen Klassifikation der subaxialen HWS-Verletzungen wird die Indikation für ein konservatives oder operatives Vorgehen gestellt.

- Impaktionsbrüche (Typ A1 sowie Spaltbrüche (Typ A2) werden i.d.R. konservativ behandelt. Eine klare, evidenzbasierte Trennlinie zwischen konservativer und operativer Behandlung gibt es nicht.

- Bei allen Verletzungen des Typs B und $C$ ist die operative Therapie indiziert.

- Der ventrale Zugang bei subaxialen HWS-Verletzungen verläuft in anatomischen Strukturen, weichteilschonend und ist als Standardzugang etabliert. Auch im Falle von Luxationsverletzungen mit der Notwendigkeit zur Reposition hat sich dieses Vorgehen bewährt.

- Ein kombiniertes ventrodorsales Vorgehen ist bei hochinstabilen Distraktions- und Rotationsverletzungen erforderlich. Hinweise hierfür sind luxierte Wirbelgelenke.

- Bei den Operationsverfahren kommen winkelstabile Implantate (Schrauben-Platten-Systeme oder Schrauben-Stab-Systeme) zur Anwendung.

\section{Korrespondenzadresse}

\section{Dr. S. Matschke}

Klinik für Unfallchirurgie und Orthopädie BG Unfallklinik Ludwigshafen

Ludwig-Guttmann-Str. 13

67071 Ludwigshafen

stefan.matschke@bgu-ludwigshafen.de

\section{Einhaltung ethischer Richtlinien}

Interessenkonflikt. S. Matschke, P. Krämer, K. Wendl und P.A. Grützner geben an, dass kein Interessenkonflikt besteht.

Dieser Beitrag beinhaltet keine Studien an Menschen oder Tieren.
The supplement containing this article is not sponsored by industry.

\section{Literatur}

1. Allen BLJ, Ferguson RL, Lehmann TR et al (1982) A mechanistic classification of closed, indirect fractures and dislocations of the lower cervical spine. Spine 7:1-27

2. Cloward RB (1958) The anterior approach for removel of ruptured discs. J Neurosurg 15:602-617

3. Cornefjord M, Alemany C, Olerud C (2005) Posterior fixation of subaxial cervical spine fractures in patients with ankylosing spondylitis. Eur Spine J 14:401-408

4. Goldberg W, Mueller C, Panacek E et al (2001) Distribution and patterns of blunt traumatic cervical spine injury. Ann Emerg Med 38:17-21

5. Goradia D, Linnau KF, Cohen WA, Mirza S, Hallam DK, Blackmore CC (2007) Correlation of MR imaging findings with intraoperative findings after cervical spine trauma. AJNR Am J Neuroradiol 28(2):209-215

6. Greenbaum J, Walters N, Levy PD (2009) An evidenced-based approach to radiographic assessment of cervical spine injuries in the emergency department. J Emerg Med 36:64-71

7. Harris JH, Edeiken-Monroe B, Kopaniky DR (1986) A practical classification of acute cervical spine injuries. Orthop Clin North Am 17(1):15-30

8. Holdsworth F (1970) Fractures, dislocations, and fracture-dislocations of the spine. J Bone Joint Sur Am 52(8):1534-1551

9. National Spinal Cord Injury Association Resource Center (2013) www.sci-info-pages.com/factsheets. html. Zugegriffen: 12. Apr. 2013

10. Lehmann W, Briem D, Blauth M et al (2005) Biomechanicalcomparison of anterior cervical spine locked and unlocked plate- fixation systems. Eur Spine J 243-249

11. Magerl F, Aebi M, Gertzbein SD et al (1994) A comprehensive classification of thoracic and lumbar injuries. Eur Spine J 3:184-201

12. Morishita Y et al (2008) The effects of degenerative changes in the functional spinal unit on the kinematics of the cervical spine. Spine (Philla Pa 1976) 33(6):E178-E182

13. Pitzen T, Wilke HJ, Caspar C et al (1999) Evaluation of a new monocortical screw for anterior cervical fusion and plating by a combined biomechanical and clinical study. Eur Spine J 8 
14. Reindl R, Ouellet J, Harvey EJ et al (2006) Anterior reduction for cervical spine dislocation. Spine 31:648-652

15. Sanchez B, Waxman K, Jones T, Conner S, Chang R, Becerra S (2005) Cervical spine clearance in blunt trauma: evaluation of a computed tomographybased protocol. J Trauma 59:179-183

16. Schleicher P, Scholz M, Schnake K et al (2008) Standarddiagnostik und Management von subaxialen HWS-Verletzungen. Trauma Berufskrankh 10(52):175-181

17. Schnacke KJ, Audigè, L, Reinhold M, Bellabarba M, Toyone T, Öner C (2013) Entwicklung und Validierung einer modifizierten Magerl-Klassifikation für subaxiale HWS-Frakturen. Meeting abstract Deutscher Kongress für Orthopädie und Unfallchirurgie, Berlin, 22.-25.10.2013

18. Sekhon LH, Fehlings MG (2001). Epidemiology, demographics and pathophysiology of acute spinal cord injury. Spine 26:S2-S12

19. Ulrich C, Bühren V (2006) Verletzungen der Halswirbelsäule. Orthopädie und Unfallchirurgie up2date 1:415-441

20. Vaccaro AR, Hulbert RJ, Patel AA, Fisher C, Dvorak M, Lehman RA et al (2007) The subaxial cervical spine injury classification system: a novel approach to recognize the importance of morphology, neurology, and integrity of the disco-ligamentous complex. Spine 32(21):2365-2374 